\title{
The Quantitative Relationship Between Physical Examinations and the Nerve Conduction of the Carpal Tunnel Syndrome in Patients With and Without a Diabetic Polyneuropathy
}

\author{
Dae Ho Jeong, MD, Chang Hwan Kim, MD \\ Department of Physical and Rehabilitation Medicine, Inha University School of Medicine, Incheon, Korea
}

\begin{abstract}
Objective To understand the quantitative correlation between the clinical severity and physical examinations along with the electrodiagnostic findings by subjects with carpal tunnel syndrome (CTS) and also the influence of diabetic polyneuropathy (DPN) on physical examinations by subjects with CTS.

Methods Among 200 patients suffering from hand tingling sensations, 68 patients were diagnosed with CTS on at least one hand by nerve conduction tests. Therefore, the Phalen test (PT), hand elevation test (HET), Tinel sign (TS) results were recorded on both hands. The physical examination grades were compared with the electrophysiological CTS grades in 126 hands of 68 patients. Also the comorbidity effect of DPN to CTS was evaluated. For the evaluation of the severity correlations between CTS, PT, HET, and TS, the Spearman analysis was used. An attempt was started to create a formula which could depict the electrophysiological severity of CTS. Results Out of the 68 tested subjects, 31 were diagnosed with both DPN and CTS, and 37 with CTS only. Both PT and HET correlated well with the severity of CTS where the correlation of PT was higher than that of HET. The formula were the motor distal latency $(\mathrm{MDL})=(72.4-\mathrm{PT}) / 5.3$ and $\mathrm{MDL}=(76-\mathrm{HET}) / 7.2$. Both PT and HET showed in the presence of DPN a relatively higher relation with CTS without significance.

Conclusion PT and HET would be useful screening tools for the diagnosis and treatment of CTS as the grade of PT and HET present the severity of CTS well. During this study, a formula was created expecting the severity of nerve conduction study with PT and HET through the time domain value of physical examinations.
\end{abstract}

Keywords Carpal tunnel syndrome, Diabetic polyneuropathy, Physical examination, Electrodiagnosis

Received June 25, 2013; Accepted December 3, 2013

Corresponding author: Chang Hwan Kim

Department of Physical and Rehabilitation Medicine, Inha University School of Medicine, 27 Inhang-ro, Jung-gu, Incheon 400-711, Korea Tel: +82-32-890-2480, Fax: +82-32-890-3445, E-mail: jacob.kim@inha. ac.kr

(c) This is an open-access article distributed under the terms of the Creative Commons Attribution Non-Commercial License (http://creativecommons. org/licenses/by-nc/3.0) which permits unrestricted noncommercial use, distribution, and reproduction in any medium, provided the original work is properly cited.

Copyright $\odot 2014$ by Korean Academy of Rehabilitation Medicine

\section{INTRODUCTION}

Carpal tunnel syndrome (CTS) is the most common nerve entrapment disorder and the clinical symptoms were first reported by James Paget in 1863. As standard diagnostic method of CTS the electrophysiological study (nerve conduction study [NCS]) was established. Magnetic resonance image and ultrasonography are the con- 
structional diagnostic tools for CTS.

Many etiologies are known for subjects with hand tingling sensations, for example, a stroke in the central nervous system or the cervical radiculopathy (herniated nucleus pulposus), a brachial plexopathy, CTS, and polyneuropathies in the peripheral nervous system.

Diabetic polyneuropathy (DPN) is a frequent cause of hand tingling sensations. It can be diagnosed using an electrodiagnostic test where the length dependent patterns exhibit the abnormalities [1]. A correct diagnosis is critical for appropriate treatments [2-5].

The diagnostic physical examinations for the CTS screening are the Phalen test (PT), hand elevation test (HET), and Tinel sign (TS). Among these, PT and HET are reported to be highly correlated with the electrophysiological diagnosis of CTS $[6,7]$. However, previous studies were focused on qualitative physical examinations only. It is hard to elucidate the exact severity of electrophysiological grading during a physical examination even though its importance is well known. Therefore, we focused on two questions in this study.

First, the formula was studied which could yield the electrophysiological severity of CTS based on physical examinations. Further studies are needed to explore the time domain of quantitative physical examinations. Second, based on the fact that diabetes mellitus has been regarded as an underlying cause of CTS, the influence of diabetic neuropathy on physical examinations of CTS was studied. Further studies are needed to reveal the differences between diabetic and non-diabetic neuropathic patients to compare the severity between the findings in physical exams and in NCSs.

\section{MATERIALS AND METHODS}

\section{Subjects}

A prospective study with 200 patients suffering from hand tingling sensations was conducted. Out of the 200 patients 68 patients, 18 male and 50 female between 31 and 80 years with a mean age $55.0 \pm 11.7$ years were selected. Mid-forties and those in their fifties accounted for half of the whole population. Thirty-one patients suffered from diabetes with diabetic neuropathy (DPN+CTS) and 37 had no diabetes (CTS only).

Patients with a history of any other systemic disease or a history of cervical and lumbar radiculopathy were ex- cluded. Patients with diabetic neuropathy without CTS were excluded. All patients who consented to participate in this study were informed about the electrodiagnostic study and this protocol, which was approved by the Institutional Review Board of our hospital.

\section{Methods}

All 68 patients underwent a NCS and physical examinations including a PT, HET, and TS evaluation. One hundred thirty-six hands (including 9 normal hands on NCS) were analyzed. The TS was recorded as positive or negative. The PT and HET were performed over 60 seconds, and if a patient felt a tingling sense, the test was stopped and the time was recorded. The time data for the patients who had no tingling sensation after 60 seconds via the PT and HETs were entered as 61 seconds. Further, the time taken for PT and HET was graded: 1-20 seconds as grade $1 ; 21-40$ seconds as grade 2; 41-60 seconds as grade 3; and longer than 60 seconds as grade 4 .

For the NCS was used the Keypoint (Dantec, Skovlunde, Denmark). The skin temperature of patients was regulated to above $32^{\circ} \mathrm{C}$. The antidromic technique was used for the sensory nerve conduction test through the median, ulnar, superficial peroneal and sural nerves. Also the median, ulnar, deep peroneal and tibial nerves were evaluated for motor NCS. The amplitude was recorded from baseline to peak. The filtering frequency was $20 \mathrm{~Hz}$ to $10 \mathrm{kHz}$ for the motor nerves and $20 \mathrm{~Hz}$ to $2 \mathrm{kHz}$ for the sensory nerves. The mean value of 10 results was taken for the sensory NCS and the supramaximal stimulation for the minimal F-M latencies was used more than 10 times of the stimulations. For the diagnosis of DPN were used the electrodiagnostic values of median, ulnar, peroneal, sural and tibial nerves. This diagnosis procedure based on our laboratory's standard which was modified from a trial research group responsible for a study about diabetes control and complications in 1995 [8,9]. Further, more than 3 abnormal values in more than 2 different nerves were counted as minimal requirement for diabetic neuropathy (Table 1).

The diagnosis of CTS was based on onset (motor nerve) or peak (sensory) latency, amplitudes and the conduction velocity of the sensory \& motor median nerves (segmental conduction on median nerve or comparison with other nerves) [10]. Then, the Bland's classification [11] based classification system was applied on motor laten- 
Table 1. Electrodiagnostic criteria for diabetic neuropathy

\begin{tabular}{|c|c|}
\hline Nerve & Values \\
\hline \multicolumn{2}{|l|}{ Median motor nerve } \\
\hline Latency (ms) & $>4.0$ \\
\hline Amplitude (mV) & $<5.0$ \\
\hline Conduction velocity $(\mathrm{m} / \mathrm{s})$ & $<49.0$ \\
\hline \multicolumn{2}{|l|}{ Median sensory nerve } \\
\hline Latency (ms) & $>3.5$ \\
\hline Amplitude $(\mu \mathrm{V})$ & $<10.0$ \\
\hline \multicolumn{2}{|l|}{ Ulnar motor nerve } \\
\hline Latency (ms) & $>3.8$ \\
\hline Amplitude (mV) & $<5.0$ \\
\hline Conduction velocity $(\mathrm{m} / \mathrm{s})$ & $<49.0$ \\
\hline Minimal F-M latency (ms) & $<24.8$ \\
\hline \multicolumn{2}{|l|}{ Ulnar sensory nerve } \\
\hline Latency (ms) & $>3.4$ \\
\hline Amplitude $(\mu \mathrm{V})$ & $<7.5$ \\
\hline \multicolumn{2}{|l|}{ Peroneal motor nerve } \\
\hline Latency (ms) & $>4.5$ \\
\hline Amplitude (mV) & $<1.0$ \\
\hline Conduction velocity $(\mathrm{m} / \mathrm{s})$ & $<40.0$ \\
\hline Minimal F-M latency (ms) & $>45$ or absent \\
\hline \multicolumn{2}{|c|}{ Superficial peroneal sensory nerve } \\
\hline Latency (ms) & $>3.5$ \\
\hline Amplitude $(\mu \mathrm{V})$ & $<3.7$ \\
\hline \multicolumn{2}{|l|}{ Tibial motor nerve } \\
\hline Latency (ms) & $>5.0$ \\
\hline Amplitude $(\mu \mathrm{V})$ & $<5.0$ \\
\hline Conduction velocity $(\mathrm{m} / \mathrm{s})$ & $<40.0$ \\
\hline Minimal F-M latency (ms) & $>45.3$ or absent \\
\hline \multicolumn{2}{|l|}{ Sural sensory nerve } \\
\hline Latency (ms) & $>3.5$ \\
\hline Amplitude $(\mu \mathrm{V})$ & $<5.0$ \\
\hline
\end{tabular}

cy, amplitude, and F-M latency. The classifications were grade 1 (electrodiagnostic grade [EG] 1) for very mild CTS and mild CTS, grade 2 (EG2) for moderately severe CTS and severe CTS, grade 3 (EG3) for very severe CTS and extremely severe CTS (Table 2).

In diabetic patients, sensory latency of median nerve comparisons with that of ulnar nerve or definite conduction block under carpal tunnel, or relative distal median motor latency delay compared to ulnar motor distal latency, were another consideration for CTS diagnosis $[12,13]$. A sensory latency of the median nerve compared to that of the ulnar nerve, a definite conduction block under the carpal tunnel or a relative distal median motor latency delay compared to the ulnar motor distal latency could be another consideration for the CTS diagnosis in patients with diabetes $[12,13]$.

To assess the association between the severity of CTS and the observed time of physical examinations the Spearman rank correlation coefficient was used. To compare the severity of DPN and CTS, the t-test was used. To compare only the CTS patients with DPN and CTS, the Pearson chi-square test was used. For the analysis of electrodiagnostic values and the physical examinations of CTS, the multiple linear regression analysis was used. As statistical software was used the SPSS ver. 18.0 (SPSS Inc., Chicago, IL, USA). Null hypotheses of no difference were rejected if $\mathrm{p}$-values were less than 0.05 .

\section{RESULTS}

\section{Severity of CTS}

The grades of electrophysiologic and physical examinations were arranged in Table 3. Among 136 hands of 68 patients, 77 hands were EG1, 37 hands were EG2, and 13 hands were EG3. Among 68 patients, 9 patients showed CTS in only one hand. The hand without CTS was defined as EG0 (Table 3).

Table 2. Modified electrodiagnostic grade of carpal tunnel syndrome

\begin{tabular}{cll}
\hline Grade & Severity & \multicolumn{1}{c}{ Nerve conduction study } \\
\hline 0 & Normal & No neurophysiological abnormality \\
1 & Mild & Detected only in two sensitive tests or Motor terminal latency $<4.5 \mathrm{~ms}$ \\
2 & Moderately & Motor terminal latency $4.5-6.5 \mathrm{~ms}$ \\
3 & Severe & Motor terminal latency $>6.5 \mathrm{~ms}$ or Surface motor potential from APB $<0.2 \mathrm{mV}$, peak-to-peak \\
\hline
\end{tabular}

Modified form Bland's classification [11] of carpal tunnel syndrome.

$\mathrm{APB}$, abductor pollicis brevis. 


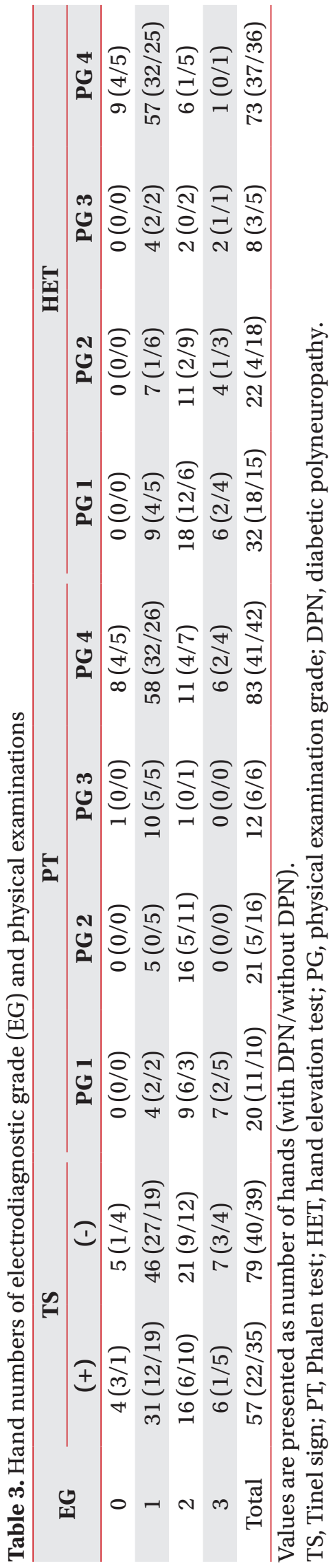

Physical examination

Tinel sign

Among 9 hands of EG0, 4 hands were positive (+) and 5 hands negative (-) in TS. In 77 hands of EG1, 31 hands were (+) and 46 hands were (-). In 37 hands of EG2, 16 hands were (+) and 21 hands were (-). In 13 hands of EG3, 6 hands were (+) and 7 hands were (-) (Table 3).

\section{Phalen test}

In EG0, all 9 hands were classified into physical examination grade 4 (PG4). In 77 hands of EG1, 5 hands were PG1, 4 hands were PG2, 8 hands were PG3, and 60 hands were PG4. In 37 hands of EG2, 9 hands were PG1, 15 hands were PG2, 2 hands were PG3, and 11 hands were PG4. In 13 hands of EG3, 6 hands were PG1, 7 hands were PG4 (Table 3).

\section{Hand elevation test}

All 9 hands at EG0 were found to be PG4. In 77 hands of EG1, 10 hands were PG1, 6 hands were PG2, 4 hands were PG3, and 57 hands were PG4. In 37 hands of EG2, 17 hands were PG1, 12 hands were PG2, 2 hands were PG3, and 6 hands were PG4. In 13 hands of EG3, 5 hands were PG1, 4 hands were PG2, 3 hands were PG3, and 1 hand was PG4 (Table 3).

Correlation between CTS severity (EG) and physical examination grade

Phalen test

Between the PT and the electrodiagnostic severity of CTS, a meaningful correlation was observed with $\mathrm{r}=0.700$ $(\mathrm{p}<0.05)$. The values with separation of DPN comorbidity also yielded a similar correlation. The patients with only CTS showed an $\mathrm{r}=0.683(\mathrm{p}<0.01)$ and CTS with comorbid DPN showed an $r=0.719(p<0.01)($ Table 4$)$.

\section{Hand elevation test}

Between the HET and the electrodiagnostic severity of CTS, a meaningful correlation was observed with $\mathrm{r}=0.635$ $(\mathrm{p}<0.01)$. The values with separation of DPN comorbidity also yielded a similar correlation. The patients with only CTS showed an $\mathrm{r}=0.578(\mathrm{p}<0.01)$ and CTS with comorbid DPN showed an $\mathrm{r}=0.708(\mathrm{p}<0.01)($ Table 4$)$. 
Table 4. Statistical analysis of 136 hands (total)

\begin{tabular}{lccc}
\hline \multirow{2}{*}{ EG } & \multicolumn{1}{c}{$\mathbf{r}$} \\
\cline { 2 - 4 } Total & TS & PT & HET \\
\hline CTS with DPN & 0.083 & $-0.700^{*}$ & $-0.635^{*}$ \\
CTS without DPN & 0.094 & $-0.719^{*}$ & $-0.708^{*}$ \\
\hline
\end{tabular}

EG, electrodiagnostic grade; TS, Tinel sign; PT, Phalen test; HET, hand elevation test; DPN, diabetic polyneuropathy. ${ }^{*} \mathrm{p}<0.01$.

Table 5. Relationship of DPN on physical examination of carpal tunnel syndrome

\begin{tabular}{|c|c|c|c|c|c|c|}
\hline & \multicolumn{2}{|c|}{ TS } & \multicolumn{2}{|c|}{ PT* } & \multicolumn{2}{|c|}{ HET* } \\
\hline & DPN (+) & DPN (-) & DPN (+) & DPN (-) & DPN (+) & DPN (-) \\
\hline Positive & $22 / 57(38.6)$ & $35 / 57(61.4)$ & $21 / 53(39.6)$ & $32 / 53(60.4)$ & $25 / 63(39.7)$ & $38 / 63(60.3)$ \\
\hline Negative & $40 / 79(50.6)$ & $39 / 79(49.4)$ & $41 / 83(49.4)$ & $42 / 83(50.6)$ & $38 / 73(52.1)$ & $36 / 73(49.3)$ \\
\hline
\end{tabular}

Values are presented as number (\%).

TS, Tinel sign; PT, Phalen test; HET, hand elevation test; DPN, diabetic polyneuropathy.

${ }^{*} \mathrm{p}>0.05$ by chi-square test.

Formula for expectation of electrodiagnostic severity

Phalen test and motor distal latency

The PT and the motor distal latency (MDL) were observed between the linear regression analyses. A formula was obtained from PT $=67.0-(4.0 \times \mathrm{MDL})$. In CTS with DPN group, a formula was obtained from PT=68.5(4.2×MDL). Separately, the in CTS without DPN group, a formula was obtained from $\mathrm{PT}=65.4-(3.8 \times \mathrm{MDL})$.

\section{Hand elevation test and motor distal latency}

The relationship between HET and MDL was observed between the linear regression analyses. A formula was obtained from HET $=71.9-(6.2 \times \mathrm{MDL})$. In CTS with DPN group, a formula was obtained from HET $=72.9-$ (6.5×MDL). Separately in the CTS without DPN group, a formula was obtained from HET=70.9-(5.8×MDL).

\section{Effect of DPN on the physical examination of CTS}

The effect of DPN on the physical examination of CTS has been studied. The PT and HET showed a relative higher presentation in patients with DPN and the inversely TS was more frequent. However, these differences were statistically insignificant (Table 5).

\section{DISCUSSION}

The usefulness of the physical examinations by CTS is well known and proved in former studies. As screening tools in the diagnosis of CTS, the TS, PT, and HET are the most common methods. In previous studies, the sensitivity and specificity of the PT, HET, and TS in CTS were often evaluated. Bruske et al. [14] reported the sensitivity of the PT ranged between $42 \%-85 \%$, and between $38 \%-100 \%$ in TS. In other studies, it was reported also the specificity of PT ranged from $54 \%-98 \%$, and between $55 \%-100 \%$ in TS. On the contrary, Mondelli et al. [15] reported that traditional provocative tests, such as PT, TS, and HET, had only limited value or were without value for the distinction of patients with and without CTS.

Only a small number of studies were conducted yet regarding the prediction values of NCS in the median nerve under use of physical examinations like the PT and HET in CTS patients. In the present study, the TS did not have predictive values, but the PT and HET showed significant correlation and predictive values. In this study, patients with electrodiagnostically mild CTS (EG1) showed no abnormality in the physical examination (PT 74\%, HET $78 \%$ ), although physical examinations cannot be exactly the same.

A formula was created for the expectation of electrodiagnostic severity in CTS patients with or without DPN. In both cases, the formula showed a little difference without statistical significance.

Mondelli et al. [15] reported positive physical signs in 
the polyneuropathy group $38.2 \%$ in PT, $38.6 \%$ in TS. This study can suggest the possibility that physical signs would be affected by neuropathy only. In the present study, the CTS group with DPN showed relatively higher positive physical findings than the CTS group without DPN.

The study showed a few limitations. 1) The tenor of this study was to understand the effect of DPN on symptomatic CTS. CTS patients with DPN and CTS only patients were enrolled. Thus, other patients, such as patients with DPN but without CTS or patients with a "normal" diabetes mellitus, were not enrolled. This would cause a sampling bias of the whole picture of neuropathic symptom evaluations. 2) In this study, there are too many bilateral CTS patients (90\%) compared to a normal CTS population with about $50 \%$. Therefore, this high percentage of bilateral symmetry of CTS in this study may cause some sampling bias, such as certain characteristics of patients [16].3) Also, there were too many normal PT (64\%) and normal HET (44\%) patients which means there could be bias in the analysis procedure and our formula. 4) Finally, the number of mild CTS patients was larger and the study may fit with early symptomatic patients more. However, patients with severe CTS do not show any symptoms or physical signs sometimes due to severe sensory nerve degeneration, and thus the presented model cannot predict electrodiagnostic values.

In this study, patients with CTS with/without DPN were compared separately. Both groups correlated well with the PG grading. Also, the group with CTS and DPN yielded a greater correlation than the group with CTS without DPN, which means further studies on this issue would be required.

The prediction values of NCS in the regression analysis of NCS with PT and HET showed meaningful results. This leads to the prediction of a NCS value through physical examinations. In addition, since the postoperative neurophysiologic changes cannot be measured every time, it can be used as a useful indicator for the recovery after a carpal tunnel release.

Given the above results and as shown in previous studies, HET and PT in relation to CTS were useful and accurate physical examinations. The TS showed less correlation, and could be considered as a limited value therefore. Also, these results showed graded physical examinations and severity classification based on NCS values of CTS are related closely.
The prediction of CTS and NCS values using the PT and HET were evaluated along with these results. The formulas achieved in the results above are rough but can be considered as valuable. Screening tests are possible and a prediction can be made regarding the NCS results based on these formulas without electrodiagnostic tests and only with simple physical examinations in the outpatient clinic. Therefore, the severity grading of CTS and a suggestion of treatment options became feasible as well.

\section{CONFLICT OF INTEREST}

No potential conflict of interest relevant to this article was reported.

\section{REFERENCES}

1. Heller L, Ring H, Costeff H, Solzi P. Evaluation of Tinel's and Phalen's signs in diagnosis of the carpal tunnel syndrome. Eur Neurol 1986;25:40-2.

2. Bracker MD, Ralph LP. The numb arm and hand. Am Fam Physician 1995;51:103-16.

3. Sato Y, Honda Y, Iwamoto J, Kanoko T, Satoh K. Amelioration by mecobalamin of subclinical carpal tunnel syndrome involving unaffected limbs in stroke patients. J Neurol Sci 2005;231:13-8.

4. Lo SF, Chou LW, Meng NH, Chen FF, Juan TT, Ho WC, et al. Clinical characteristics and electrodiagnostic features in patients with carpal tunnel syndrome, double crush syndrome, and cervical radiculopathy. Rheumatol Int 2012;32:1257-63.

5. Morgan G, Wilbourn AJ. Cervical radiculopathy and coexisting distal entrapment neuropathies: doublecrush syndromes? Neurology 1998;50:78-83.

6. Ahn DS. Hand elevation: a new test for carpal tunnel syndrome. Ann Plast Surg 2001;46:120-4.

7. Gellman H, Gelberman RH, Tan AM, Botte MJ. Carpal tunnel syndrome: an evaluation of the provocative diagnostic tests. J Bone Joint Surg Am 1986;68:735-7.

8. Diabetes Control and Complications Trial Research Group. The effect of intensive diabetes therapy on the development and progression of neuropathy. Ann Intern Med 1995;122:561-8.

9. Han EY, Kim CH. Reproducibility of nerve conduction study parameters: a comparison of normal and diabetic patients with neuropathy. Ann Rehabil Med 
2007;31:699-704.

10. Imaoka H, Yorifuji S, Takahashi M, Nakamura Y, Kitaguchi $\mathrm{M}$, Tarui S. Improved inching method for the diagnosis and prognosis of carpal tunnel syndrome. Muscle Nerve 1992;15:318-24.

11. Bland JD. A neurophysiological grading scale for carpal tunnel syndrome. Muscle Nerve 2000;23:1280-3.

12. Felsenthal G. Median and ulnar distal motor and sensory latencies in the same normal subject. Arch Phys Med Rehabil 1977;58:297-302.

13. Kwon HK, Kim L, Park YK, Lee HJ. Frequency of carpal tunnel syndrome according to the severity of diabetic neuropathy. J Korean Acad Rehabil Med 2005;29:272-5.

14. Bruske J, Bednarski M, Grzelec H, Zyluk A. The usefulness of the Phalen test and the Hoffmann-Tinel sign in the diagnosis of carpal tunnel syndrome. Acta Orthop Belg 2002;68:141-5.

15. Mondelli M, Passero S, Giannini F. Provocative tests in different stages of carpal tunnel syndrome. Clin Neurol Neurosurg 2001;103:178-83.

16. Felsenthal G, Spindler H. Palmar conduction time of median and ulnar nerves of normal subjects and patients with carpal tunnel syndrome. Am J Phys Med 1979;58:131-8. 